\title{
Finite-Time Spacecraft's Soft Landing on Asteroids Using PD and Nonsingular Terminal Sliding Mode Control
}

\author{
Keping Liu, Fengxia Liu, Shenquan Wang, and Yuanchun Li \\ College of Electrical and Electronic Engineering, Changchun University of Technology, Changchun 130000, China \\ Correspondence should be addressed to Yuanchun Li; liyc@mail.ccut.edu.cn
}

Received 20 June 2014; Accepted 11 September 2014

Academic Editor: Carlo Cosentino

Copyright (C) 2015 Keping Liu et al. This is an open access article distributed under the Creative Commons Attribution License, which permits unrestricted use, distribution, and reproduction in any medium, provided the original work is properly cited.

\begin{abstract}
This paper presents a continuous control law of probe, which consists of PD (proportional-derivative) controller and nonsingular terminal sliding mode controller for probe descending and landing phases, respectively, in the case of the asteroid irregular shape and low gravity. The probe dynamic model is deduced in the landing site coordinate system firstly. Then the reference trajectory based on optimal polynomial in open-loop state is designed, with the suboptimal fuel consumption. Taking into account different characteristics of phases, PD controller and nonsingular terminal sliding mode controller can be employed in the descending phase and the landing phase, respectively, to track the designed reference trajectory. The controller which used the corresponding control methods can meet the motion characteristics and requirements of each stage. Finally simulation experiments are carried out to demonstrate the effectiveness of the proposed method, which can ensure the safe landing of probe and achieve continuous control.
\end{abstract}

\section{Introduction}

Exploration of comets and asteroids is one of the most complex missions in the future [1]. Asteroids exploration has been identified as a significant space mission; asteroids are believed to exist in the early stage of the solar system and be helpful to study solar system [2]. Spacecrafts are required to achieve a soft land on the asteroids, which use certain devices, control the orbit to gradually reduce landing speed, and finally landing without damage to the surface of the ground.

On February 12, 2001, Near Earth Asteroid Rendezvous (NEAR) probe successfully landed on the Eros asteroid $[3,4]$. On May 9, 2003, MUSES-C probe was launched and took samples of rock to earth at first time [5]. On December 13, 2012, China's Chang'e II flew over international number 4179 Toutatis asteroid with the relative velocity of $10.73 \mathrm{~km} / \mathrm{s}$.

Due to the irregular shape, the small size, and the unstructured uncertainties of asteroid, a system with guidance, navigation, and control (GNC) is needed for asteroid landing safely and precisely [6]. The control of GNC has received tremendous research interest in the past years, and a number of scholars focus attention on this subject [7-9]. A landing autonomous optical navigation scheme of the small body based on the landing site feature extraction and tracking technology was proposed in the literature [7]. Reference [8] studied an autonomous navigation algorithm and a control strategy based on sliding mode variable structure guidance was provided via the optical information and Kalman filter. A self-guided method based on optical camera and laser radar was proposed in [9], in which the sliding mode variable structure control was used to make sure the probe landed safely. The researchers also do some studies on reducing fuel consumption of the probe [10-13]. The fuel optimal polynomial nominal trajectory and the control method based on PD plus PWPF (pulse-width pulse-frequency) were designed in [10]. The new algorithm was developed in Matlab based on the performance database (PDB) to design optimisation trajectory and obtain the best aircraft fuel efficiency and the reduction in fuel consumption on aircraft [11]. A hybrid actuation control concept, a fuzzy logic proportional-integralderivative (PID) plus a conventional on-off controller, was described, and the controller would behave as a switch between the shape memory alloys (SMA) cooling and heating phases, situations where the output current was $0 \mathrm{~A}$ or was controlled by the fuzzy logic controller (FLC) $[12,13]$. 
The landing process of probe was transformed into tracking the reference trajectory and the variable structure control was proposed for the probe control problem in literatures $[14,15]$. The probe landing at a specific location as free falling and the tracking error were analyzed in detail [16]. The polyhedron dynamic model was set up based on threedimensional scattered points on surface of the small bodies [17]. To reduce the structured and unstructured uncertainties of the probe, a new control scheme based on two adaptive neural controllers was proposed with good performance [18]. Finite-time control method, which can tackle the probe convergence fast, was proposed to improve the stability and robustness of system [19].

Summarizing the above discussion, the continuous control during drop and landing phase needs further investigation. This paper investigates characteristics of descending and landing phases, and PD controller and nonsingular terminal sliding mode controller are designed. In the descending phase, PD controller is adopted with the purpose of reducing the horizontal component of the velocity and position. In the landing phase, the positions of $x$ - and $y$-axes approach zeros quickly and probe is over landing point vertically. Nonsingular terminal sliding mode control method based on exponential reaching law can avoid the singular problems on sliding surface, reduce the adjusting time, and guarantee the rapidity and stability of probe landing. The remaining of the paper is organized as follows. In Section 2, probe dynamic model is described in the landing site coordinate system, taking into account the low gravity of small body and main factors in the orbit. In Section 3, the reference trajectory with the suboptimal fuel consumption is designed, and PD controller and nonsingular terminal sliding mode controller are proposed to track reference trajectory and make probe land on the surface of small body quickly and safely. Simulation results are presented and discussed in Section 4. Finally, a conclusion regarding research works is provided in Section 5.

\section{Asteroid and Probe Models}

2.1. Gravity Field Model of Asteroid. The gravity model of irregular shape asteroids should be established, and the problem on the characteristic of gravitational field must be clarified. In this work, the target asteroid can be approximated as a triaxial ellipsoid $[20,21]$, and the gravitational potential energy of asteroid can be obtained with spherical harmonic series expansion method. Gravitation can be described, with the gradient of the gravitational potential function, as follows:

$$
\begin{gathered}
F_{a}=\operatorname{grad}[U(r, \theta, \phi)], \\
U(r, \theta, \phi)=\frac{\mu_{a}}{r}\left\{1+\sum_{l=1}^{n} \sum_{m=0}^{l}\left[\frac{R_{0}}{r}\right]^{l} P_{l m}(\sin \phi)\right. \\
\left.\times\left[C_{l m} \cos m \theta+S_{l m} \sin m \theta\right]\right\},
\end{gathered}
$$

where $U(r, \theta, \phi)$ denotes gravity potential; $u_{a}$ is multiplied by the product of the gravitational constant and the mass of small body; $l$ is the degree; $m$ is the order; $R_{0}$ is reference radius; $\mathbf{r}$ is the position vector from the mass center of small body to the spacecraft; $\theta$ and $\phi$ are the latitude and longitude, respectively; $C_{l m}$ and $S_{l m}$ are the coefficients of potential determined by the mass distribution within the small body; $P_{l m}(\sin \phi)$ is the associated Legendre polynomials.

$S_{l m}=0$ for all $l$ or $m, C_{l m}=0$ for $l$ or $m$ odd and while other conditions:

$$
\begin{aligned}
C_{l m}= & \frac{3(1 / 2) !(l-m) !}{a^{l} 2^{m}(l+3)(l+1)}\left(2-\delta_{0 m}\right) \\
& \times \sum_{i=0}^{\operatorname{int}((l-m) / 4)}\left(\left(a^{2}-b^{2}\right)^{(m+4 i) / 2}\right. \\
& \left.\times\left[c^{2}-\left(\frac{1}{2}\right)\left(a^{2}+b^{2}\right)\right]^{(l-m-4 i) / 2}\right) \\
& \times\left(16^{i}\left(\frac{l-m-4 i}{2}\right) !\left(\frac{m+2 i}{2}\right) ! i !\right)^{-1},
\end{aligned}
$$

where $\delta_{0 m}$ is Kronecker symbol and the value of $\delta_{0 m}$ is

$$
\delta_{0 m}= \begin{cases}0, & m=0 \\ 1, & m=1\end{cases}
$$

By expanding to fourth order of (2), some coefficients can be obtained as follows:

$$
\begin{aligned}
& C_{20}=\frac{2 c^{2}-\left(a^{2}+b^{2}\right)}{10 a^{2}}, \\
& C_{22}=\frac{a^{2}-b^{2}}{20 a^{2}}, \\
& C_{40}=3 \frac{3\left(a^{4}+b^{4}\right)+8 c^{4}+2 a^{2} b^{2}-8\left(a^{2}+b^{2}\right) c^{2}}{140 a^{4}}, \\
& C_{42}=\frac{\left(a^{2}-b^{2}\right)\left(2 c^{2}-a^{2}-b^{2}\right)}{280 a^{4}}, \\
& C_{44}=\frac{\left(a^{2}-b^{2}\right)^{2}}{2240 a^{4}},
\end{aligned}
$$

where $a, b$, and $c$ are radius parameters of the approximate axis ellipsoid.

Therefore, the asteroid gravity is obtained as follows:

$$
\begin{aligned}
U(r, \theta, \phi)=\frac{u_{a}}{r}\{1 & +\Delta\left(\frac{R_{0}}{r}\right)^{2} \\
& \left.+\Gamma\left(\frac{R_{0}}{r}\right)^{4}+O\left(r^{-5}\right)\right\},
\end{aligned}
$$




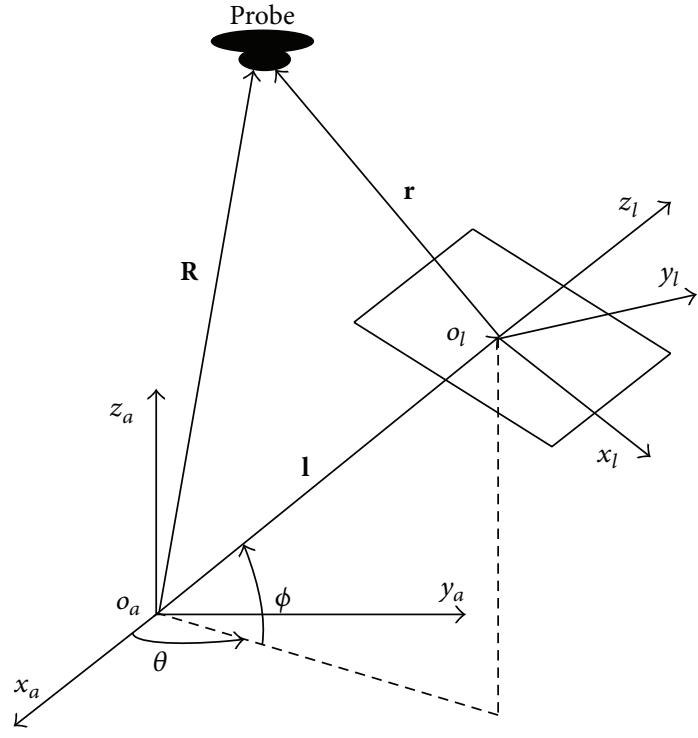

Figure 1: Coordinate systems definition.

where

$$
\begin{gathered}
\Delta=\left[\frac{1}{2} C_{20}\left(3 \sin ^{2} \phi-1\right)+3 C_{22} \cos ^{2} \phi \cos 2 \theta\right], \\
\Gamma=\left[\frac{1}{8} C_{40}\left(35 \sin ^{4} \phi-30 \sin ^{2} \phi+3\right)\right. \\
+\frac{15}{2} C_{42} \cos ^{2} \phi\left(7 \sin ^{2} \phi-1\right) \cos 2 \theta \\
\left.+105 C_{44} \cos ^{2} \phi \cos 4 \theta\right] .
\end{gathered}
$$

2.2. Probe Dynamics Model. As shown in Figure 1, the asteroid body-fixed coordinate system $o_{a} x_{a} y_{a} z_{a}$ and the probe landing site coordinate system $o_{l} x_{l} y_{l} z_{l}$ are established [22].

Let the asteroid body coordinate system be fixed on asteroid with the origin coinciding with the mass center of asteroid and the $o_{a} z_{a}$ axis coinciding with the asteroid's maximum moment of inertia, and the $o_{a} x_{a}, o_{a} y_{a}$, and $o_{a} z_{a}$ axis compose the right-handed coordinate system. As the coordinates of probe landing site is the same as the initial landing site. $\mathbf{R}$ is the position vector from the target small body mass center to the probe; $\mathbf{r}$ is the position vector from the landing point to the probe; $\mathbf{l}$ is the position vector from the mass center to the landing site.

The dynamic equations of motion for probe in the fixedbody coordinate system are given as follows:

$$
\ddot{\mathbf{R}}+2 \boldsymbol{\omega} \times \dot{\mathbf{R}}+\boldsymbol{\omega} \times(\boldsymbol{\omega} \times \mathbf{R})+\dot{\boldsymbol{\omega}} \times \mathbf{R}=\mathbf{F}+\mathbf{U}+\boldsymbol{\delta},
$$

where $\boldsymbol{\omega}$ denotes the rotation vector of the small body; $\mathbf{F}$ denotes the control acceleration; $\mathbf{U}$ denotes the gradient of the gravitational potential; $\boldsymbol{\delta}$ denotes the perturbative acceleration for no model including the solar radiation pressure and third-body gravitational perturbations

$$
\boldsymbol{\delta}=\frac{\eta \mathbf{d} \cdot \mathbf{R}}{|\mathbf{d}|^{3}}-\frac{\mu_{a}}{2 d^{3}}\left[\mathbf{R} \cdot \mathbf{R}-3\left(\frac{\mathbf{d} \cdot \mathbf{r}}{|\mathbf{d}|}\right)^{2}\right] .
$$

$\eta$ denotes the solar radiation pressure coefficient; $\mathbf{d}$ denotes the position vector of the center of mass from the asteroid to the sun.

To obtain orbit dynamic equations in the landing point coordinate, a transform matrix from landing site coordinate system to the fixed coordinate is shown as follows:

$$
\mathbf{C}_{l}^{a}=\left[\begin{array}{ccc}
\cos \theta \sin \phi & -\sin \theta & \cos \theta \cos \phi \\
\sin \theta \sin \phi & \cos \phi & \sin \theta \cos \phi \\
-\cos \phi & 0 & \sin \phi
\end{array}\right]
$$

Let $\boldsymbol{\omega} \cong \boldsymbol{\omega}_{a}=\left[\begin{array}{lll}0 & 0 & \omega_{a}\end{array}\right]^{T}$ be angular velocity vector, and the asteroid has the fixed rotation angular velocity vector of $\omega_{a}$; then, $\dot{\boldsymbol{\omega}}=0$. Therefore, the expressions of dynamic model in the landing point coordinates are given as follows:

$$
\begin{aligned}
\ddot{x}- & 2 \omega_{a} \sin \phi \dot{y}-\omega_{a}^{2} \sin ^{2} \phi x-\omega_{a}^{2} \sin \phi \cos \phi z \\
& =F_{x}+U_{x}+\delta_{x}, \\
\ddot{y}+ & 2 \omega_{a} \sin \phi \dot{x}+2 \omega_{a} \cos \phi \dot{z}-\omega_{a}^{2} y \\
& =F_{y}+U_{y}+\delta_{y}, \\
\ddot{z}- & 2 \omega_{a} \cos \phi \dot{y}-\omega_{a}^{2} \sin \phi \cos \phi x-\omega_{a}^{2} \cos ^{2} \phi z \\
& =F_{z}+U_{z}+\delta_{z},
\end{aligned}
$$

where $x, y$, and $z$ are components of probe position; $F_{x}, F_{y}$, and $F_{z}$ are components of control acceleration; $U_{x}=\partial U / \partial x$, $U_{y}=\partial U / \partial y$, and $U_{z}=\partial U / \partial z$ are components of the asteroid gravitational potential.

\section{Guidance and Control Law}

3.1. Reference Trajectory Planning. In descending phase, the primary thrust of the brake section and the low-thrust engines simultaneously brake the entire thrust of the engine braking process. The purpose is to reduce the horizontal component of the probe. In landing phase, the low-thrust engine brakes so as to adjust posture and ensure the probe lands vertically in final few hundred meters [23].

A soft landing is mentioned for probe landing to the target asteroids at small speed, which is usually less than $6 \mathrm{~m} / \mathrm{s}$ [24]. The desired descent altitude and velocity are planned in order to satisfy the requirements of soft landing on the surface of small bodies. It is assumed that the nominal trajectory is 
fuel suboptimal polynomial trajectory employed by Apollo landing lunar. The boundary condition is given by

$$
\begin{aligned}
& r_{z}(0)=z_{0}, \\
& r_{z}(\tau)=z_{n}, \\
& \dot{r}_{z}(0)=\dot{z}_{0}, \\
& \dot{r}_{z}(\tau)=0,
\end{aligned}
$$

where $z_{0}$ and $\dot{z}_{0}$ denote the initial altitude and altitude change rate, $z_{n}$ is the planned altitude, and $\tau$ is descent time.

The cubic curve to satisfy the boundary condition is given by [25]

$$
\begin{aligned}
r_{z}(t)= & z_{0}+\dot{z}_{0} t+\left(3 z_{n}-3 z_{0}-2 \dot{z}_{0} \tau\right)\left(\frac{t}{\tau}\right)^{2} \\
& +\left(2 z_{0}+\dot{z}_{0} \tau-2 z_{n}\right)\left(\frac{t}{\tau}\right)^{3} .
\end{aligned}
$$

The planned descent velocity, which is derived from the time derivative of (13), is given by

$$
\begin{aligned}
\dot{r}_{z}(t)= & \dot{z}_{0}+\left(6 z_{n}-6 z_{0}-4 \dot{z}_{0} \tau\right) \frac{t}{\tau^{2}} \\
& +\left(6 z_{0}+3 \dot{z}_{0} \tau-6 z_{n}\right) \frac{t^{2}}{\tau^{3}}
\end{aligned}
$$

By this way, $r_{x}(t), \dot{r}_{x}(t), r_{y}(t)$, and $\dot{r}_{y}(t)$ are also planned.

3.2. Continuous Control Law for Declining and Landing Phase. Define the tracking error and the velocity tracking error as $e=r-r_{d}$ and $\dot{e}=\dot{r}-\dot{r}_{d}$, where $\mathbf{r}_{\mathbf{d}}=\left[r_{x}, r_{y}, r_{z}\right]^{T}$ denotes the reference trajectory. Substituting (11) into $e$ and $\dot{e}$ yields the following equations:

$$
\begin{aligned}
& {\left[\begin{array}{c}
\dot{e}_{1} \\
\dot{e}_{2} \\
\dot{e}_{3}
\end{array}\right]=\left[\begin{array}{c}
e_{4} \\
e_{5} \\
e_{6}
\end{array}\right],} \\
& {\left[\begin{array}{c}
\dot{e}_{4} \\
\dot{e}_{5} \\
\dot{e}_{6}
\end{array}\right]=\left[\begin{array}{c}
-\ddot{r}_{x}+2 \omega_{a} \sin \phi \dot{y}+\omega_{a}^{2} \sin ^{2} \phi x+\omega_{a}^{2} \sin \phi \cos \phi z+F_{x}+U_{x}+\delta_{x} \\
-\ddot{r}_{y}-2 \omega_{a} \sin \phi \dot{x}-2 \omega_{a} \cos \phi \dot{z}+\omega_{a}^{2} y+F_{y}+U_{y}+\delta_{y} \\
-\ddot{r}_{z}+2 \omega_{a} \cos \phi \dot{y}+\omega_{a}^{2} \sin \phi \cos \phi x+\omega_{a}^{2} \cos ^{2} \phi z+F_{z}+U_{z}+\delta_{z}
\end{array}\right] .}
\end{aligned}
$$

Equation (15) can be transformed into state equations

$$
\begin{aligned}
\dot{\mathbf{e}}_{i} & =\mathbf{e}_{j}, \\
\dot{\mathbf{e}}_{j} & =\mathbf{f}_{k}+\mathbf{g}_{k}+\mathbf{u}_{k},
\end{aligned}
$$

where $\mathbf{e}_{i}$ and $\mathbf{e}_{j}(i=1,2,3 ; j=4,5,6)$ denote the system state variables; $\mathbf{f}_{k}$ denotes nonlinear dynamics of the system; $\mathbf{g}_{k}$ denotes uncertainties and disturbances and it is satisfied with $\left\|\vec{g}_{k}\right\| \leq l_{g}, l_{g}>0$; $\mathbf{u}_{k}$ denotes control law $(k=x, y, z)$;

$$
\begin{aligned}
& \mathbf{f}_{k}=\left[\begin{array}{c}
-\ddot{r}_{x}+2 \omega_{a} \sin \phi \dot{y}+\omega_{a}^{2} \sin ^{2} \phi x+\omega_{a}^{2} \sin \phi \cos \phi z+U_{x} \\
-\ddot{r}_{y}-2 \omega_{a} \sin \phi \dot{x}-2 \omega_{a} \cos \phi \dot{z}+\omega_{a}^{2} y+U_{y} \\
-\ddot{r}_{z}+2 \omega_{a} \cos \phi \dot{y}+\omega_{a}^{2} \sin \phi \cos \phi x+\omega_{a}^{2} \cos ^{2} \phi z+U_{z}
\end{array}\right] ; \\
& \mathbf{g}_{k}=\left[\begin{array}{lll}
\delta_{x} & \delta_{y} & \delta_{z}
\end{array}\right]^{T} ; \quad \mathbf{u}_{k}=\left[\begin{array}{lll}
F_{x} & F_{y} & F_{y}
\end{array}\right]^{T} .
\end{aligned}
$$

In order to get ready for soft landing, PD control laws are designed to track the reference trajectory and the desired descending velocity. The descent control laws can be designed as follows: where $k_{P x}, k_{P y}, k_{P z}, k_{D x}, k_{D y}$, and $k_{D z}$ are controller coefficients, respectively.

In landing phase, the sliding mode control is designed, which is based on the use of the following sliding surface:

$$
s_{i}=e_{i}+\frac{1}{\beta}\left(e_{j}\right)^{p / q}
$$

$$
\begin{aligned}
& F_{x}=-k_{P x} e_{1}-k_{D x} e_{4}, \\
& F_{y}=-k_{P y} e_{2}-k_{D y} e_{5}, \\
& F_{z}=-k_{P z} e_{3}-k_{D z} e_{6},
\end{aligned}
$$

where $\beta>0, p / q \in(1,2)$. By choosing the sliding surface (19), there is no negative power in system and the singular problem can be avoided [26]. 
Taking the first order derivative of $s_{i}$,

$$
\begin{aligned}
\dot{s}_{i} & =\dot{e}_{i}+\frac{1}{\beta} \frac{p}{q}\left(e_{j}\right)^{p / q-1} \dot{e}_{j} \\
& =e_{j}+\frac{1}{\beta} \frac{p}{q}\left(e_{j}\right)^{p / q-1}\left(f_{k}+g_{k}+u_{k}\right) .
\end{aligned}
$$

In order to keep the controller respond immediately, the exponential approach law is used to approach the sliding surface. By choosing

$$
\dot{s}=-\varepsilon \operatorname{sgn}(s)-k s,
$$

where $\varepsilon$ and $k$ are positive coefficients of exponential approach law.

Substituting (21) into (20), the control law is obtained as follows:

$$
u_{k}=-\rho^{-1}\left(e_{j}\right)\left[e_{j}+\rho\left(e_{j}\right)\left(f_{k}+g_{k}\right)+\varepsilon \operatorname{sgn}\left(s_{i}\right)+k s_{i}\right],
$$

where $\rho\left(e_{j}\right)=(1 / \beta)(p / q)\left(e_{j}\right)^{p / q-1}$.

As $g_{k}$ denotes uncertainties and disturbances, and it is bounded. Adding a small constant $\xi(\xi>0)$ in the control law, the new control law is obtained

$$
u_{k}=-\left[\left(\rho\left(e_{j}\right)+\xi\right)^{-1}\left(e_{j}+\varepsilon \operatorname{sgn}\left(s_{i}\right)+l_{g} \operatorname{sgn}\left(s_{i}\right)+k s_{i}\right)+f_{k}\right] .
$$

By choosing sliding mode surface (19) and the control law (23), it can ensure that the system reaches the sliding surface in finite time, and the tracking error approaches zero in finite time.

Proof. Take the first order derivative of $s_{i}$

$$
\begin{aligned}
\dot{s}_{i}= & e_{j}+\rho\left(e_{j}\right)\left(f_{k}+g_{k}+u_{k}\right) \\
= & e_{j}-\rho\left(e_{j}\right) \\
& \times\left[\left(\rho\left(e_{j}\right)+\xi\right)^{-1}\left(e_{j}+\varepsilon \operatorname{sgn}\left(s_{i}\right)+k s_{i}\right)+l_{g} \operatorname{sgn}\left(s_{i}\right)-g_{k}\right] .
\end{aligned}
$$

As long as $\xi$ is small enough, $\rho\left(e_{j}\right)\left(\rho\left(e_{j}\right)+\xi\right)^{-1}=1$ can be satisfied, and (24) can be simplified as

$$
\dot{s}_{i}=-\varepsilon \operatorname{sgn}\left(s_{i}\right)-k s_{i}-\rho\left(e_{j}\right)\left(l_{g} \operatorname{sgn}\left(s_{i}\right)-g_{k}\right) .
$$

As $e_{j} \neq 0, \beta>0$, and $p / q \in(1,2)$, then $\rho\left(e_{j}\right)>0$ and $\dot{s}_{i} \leq-\varepsilon \operatorname{sgn}\left(s_{i}\right)-k s_{i}<0$.

Note that when the probe is close to the asteroid, $g_{k}$ can be ignored

$$
\dot{s}_{i}=-\varepsilon \operatorname{sgn}\left(s_{i}\right)-k s_{i}, \quad e_{j} \neq 0 .
$$

If $e_{j}=0$, then $\rho\left(e_{j}\right)=0$ and $\dot{s}_{i}=-\varepsilon \operatorname{sgn}\left(s_{i}\right)-k s_{i}, e_{j}=0$.

Considering the control law (23) contains a term of $\left(\rho\left(e_{j}\right)+\xi\right)^{-1}$, which is complicated and needs large amount of calculation, in order to improve the probe performance of autonomic computing, (23) can be further simplified [12]

$$
u_{k}=-\beta \frac{q}{p}\left(e_{j}\right)^{2-p / q}-f_{k}-k s_{i}-\left(l_{g}+\varepsilon\right) \operatorname{sgn}\left(s_{i}\right) .
$$

By choosing sliding mode surface (16) and the control law (27), it can ensure that the system reaches the sliding surface in finite time, and the tracking error approaches zero in finite time.

Proof. Take the first order derivative of $s_{i}$

$$
\begin{aligned}
\dot{s}_{i}= & e_{j}+\rho\left(e_{j}\right)\left(f_{k}+g_{k}+u_{k}\right) \\
= & e_{j}-\rho\left(e_{j}\right) \\
& \times\left[\beta \frac{q}{p}\left(e_{j}\right)^{2-p / q}+\varepsilon \operatorname{sgn}\left(s_{i}\right)+k s_{i}+l_{g} \operatorname{sgn}\left(s_{i}\right)-g_{k}\right] \\
= & -\rho\left(e_{j}\right)\left[\varepsilon \operatorname{sgn}\left(s_{i}\right)+k s_{i}+l_{g} \operatorname{sgn}\left(s_{i}\right)-g_{k}\right] .
\end{aligned}
$$

If $e_{j} \neq 0$, then $\rho\left(e_{j}\right)>0$ and $\dot{s}_{i} \leq-\varepsilon \operatorname{sgn}\left(s_{i}\right)-k s_{i}<0$.

Lyapunov function is $V_{i}=(1 / 2) s_{i}^{T} s_{i}(i=1,2,3)$.

The first order derivative of $V_{i}(i=1,2,3)$

$$
\begin{aligned}
\dot{V}_{i} & =s_{i}^{T} \dot{s}_{i}=-s_{i}^{T} \rho\left(e_{j}\right)\left[\varepsilon \operatorname{sgn}\left(s_{i}\right)+k s_{i}+l_{g} \operatorname{sgn}\left(s_{i}\right)-g_{k}\right] \\
& \leq-s_{i}^{T}\left(\varepsilon \operatorname{sgn}\left(s_{i}\right)+k s_{i}\right)<0
\end{aligned}
$$

$\dot{V}_{i}=0$ if and only if $s_{i}=0(i=1,2,3)$, hence the tracking control law is proved to be stable.

To reduce the system chattering caused by sliding mode control, the sign function of the switching surface function is replaced by a suitable saturated function as follows:

$$
\mathbf{a}=\operatorname{sat}(\cdot)= \begin{cases}1 & s_{k}>\Delta_{k} \\ \frac{s_{k}}{\Delta_{k}} & s_{k} \leq \Delta_{k} \\ -1 & s_{k}<-\Delta_{k},\end{cases}
$$

where $\Delta_{k}>0$ is the boundary layer, $s_{k}$ is the sliding mode surface function, and $\forall_{k} \in\{1,2, \ldots, 5\}$.

Substituting (30) into (27), the final control law can be obtained as follows:

$$
u_{k}=-\beta \frac{q}{p}\left(e_{j}\right)^{2-p / q}-f_{k}-k s_{i}-\left(l_{g}+\varepsilon\right) \mathbf{a} .
$$

When the probe is close to the asteroid, the impact of $g_{k}$ is ignored. Then, $\dot{s}_{i}=-\rho\left(e_{j}\right)\left[\varepsilon \operatorname{sgn}\left(s_{i}\right)+k s_{i}\right]$. Consider

$$
\begin{gathered}
\dot{e}_{j} \leq-k s_{i}-\varepsilon, \quad s_{i}>0, \\
\dot{e}_{j} \geq-k s_{i}+\varepsilon, \quad s_{i}<0 .
\end{gathered}
$$

So

$$
\begin{array}{ll}
\dot{s}_{i} \leq-k s_{i}-\varepsilon, & s_{i}>0, \\
\dot{s}_{i} \geq-k s_{i}+\varepsilon, & s_{i}<0 .
\end{array}
$$


TABLE 1: Numerical simulation parameters.

\begin{tabular}{lcc}
\hline Parameters & Real value & Simulation parameters \\
\hline$u_{a}$ & $4.749 E-04$ & $4.800 E-04$ \\
Spin period (h) & 10.54 & 10.55 \\
$R_{0}(\mathrm{~km})$ & 1.150 & 1.148 \\
$C_{20}$ & -0.113 & -0.110 \\
$C_{22}$ & 0.0396 & 0.0397 \\
$C_{40}$ & 0.068 & 0.069 \\
$C_{42}$ & 0.0032 & 0.0031 \\
$C_{44}$ & $2.790 E-04$ & $2.780 E-04$ \\
$k_{P x}=k_{P y}=k_{P z}$ & & 0.0005 \\
$k_{D x}=k_{D y}=k_{D z}$ & & 0.05 \\
Descending initial position & & {$[3550,4050,11000]$} \\
Descending initial velocity & & {$[-2.2,1.2,-9]$} \\
Landing initial position & & {$[300,300,2000]$} \\
Landing initial velocity & & {$[20,20,20]$} \\
Landing final site & & \\
\hline
\end{tabular}

In order to avoid a greater buffeting, $\varepsilon$ is usually taken as a smaller value to ensure the probe reaches the sliding surface in the form of exponential reaching law.

Comparing two kinds of cases, if $e_{j} \neq 0$, then $\rho\left(e_{j}\right)$ is involved in the presented reaching law, which is called nonsingular terminal sliding mode control pseudoexponential approaching law (NTSMC-PEAL) and if $e_{j}=0$, then the probe reaches the sliding surface in the form of exponential reaching law, which is called nonsingular terminal sliding mode control exponential approaching law (NTSMC-EAL). NTSMC-PEAL and NTSMC-EAL, which are used in the control system for irregular asteroids, can ensure that terminal sliding mode under the condition of nonsingular uses a small control to achieve an ideal control effect.

\section{Simulation and Analysis}

Take one asteroid as the target small body, and its parameters are listed in Table $1[11,19]$. Those parameters are closely related to the descending and landing phase studied in this work. Taking into account system disturbance, some reasonable assumptions are supposed according to engineering practice. $\delta_{x}=1.5 \sin 2 t, \delta_{y}=1.6 \sin 1.5 t$, and $\delta_{z}=$ $1.4 \sin 3 t$ are chosen in simulation experiments, and the model parameters under the worst conditions are selected.

The parameters of nonsingular terminal sliding mode control are chosen as follows: $\beta=2, p / q=1.5, k=2$, and $l_{g}=0.015$.

In the descending phase and landing phase, the simulation results are shown from Figure 2 to Figure 13.

The descending trajectory curve based on PD control scheme is shown in Figure 2. The curve is smooth and the probe is prepared for landing. The position and velocity of the probe are shown in Figures 3 and 4, respectively. In Figures 5, 6 , and 7 , the position tracking curves of $x$-axis, $y$-axis, and $z$ axis are shown and demonstrate that the probe can track the reference position quickly and consistent with it completely.

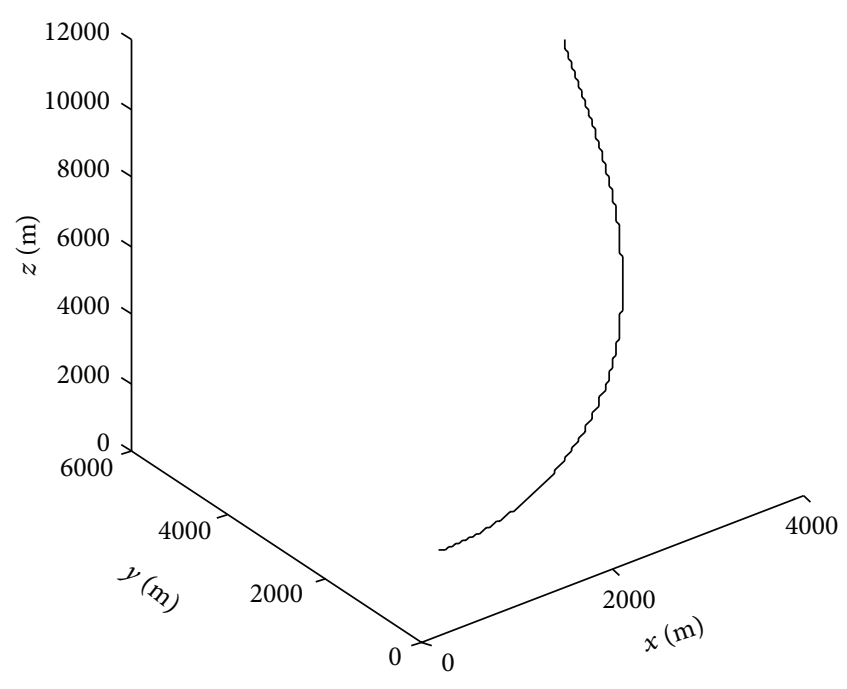

FIGURE 2: Descending trajectory curve.

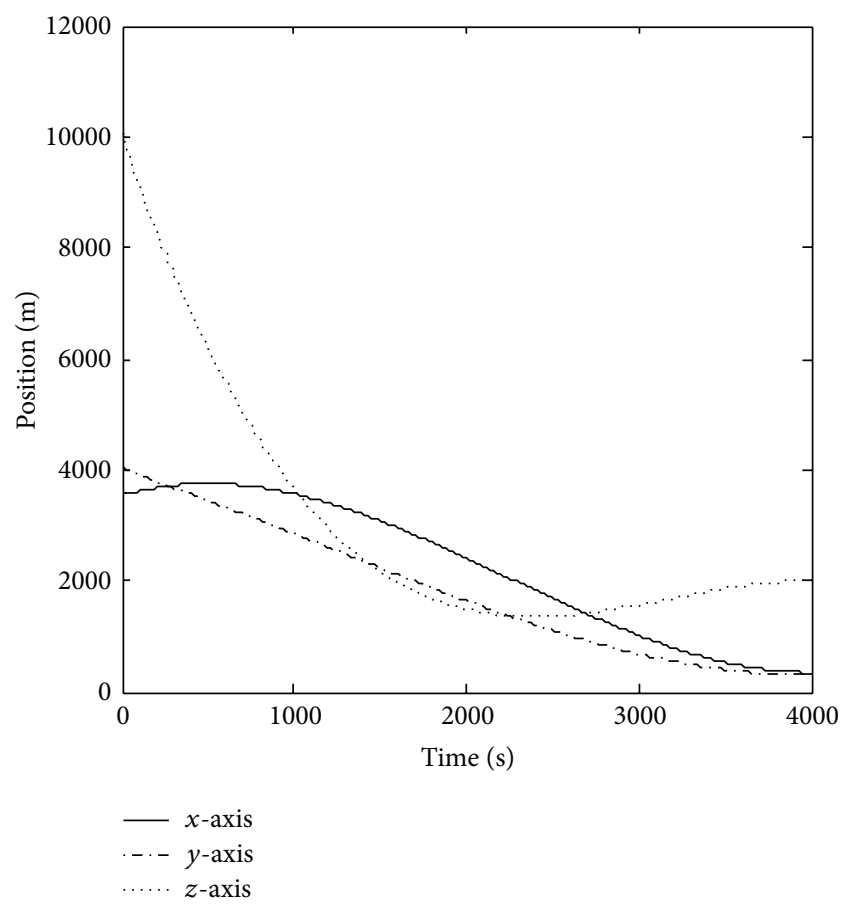

FIgURE 3: Descending position over time.

The landing trajectory curve based on NTSMC control scheme is shown in Figure 8. Comparing NTSMC with SMC, the probe based on NTSMC control scheme can track the reference curve quickly and keep the state error much more smaller, and the adjusting time is less than SMC control scheme. Moreover, the oscillation in the landing process of the probe is reduced, and the tracking error approaches zero quickly in a limited time. From Figure 9 to Figure 10, the position and velocity of the probe can be shown; moreover, the control force can be satisfied with engineering requirements. As seen in Figure 11, the position error of $z$-axis is less than $1 \mathrm{~m}$; the NTSMC control scheme can satisfy the requirement 


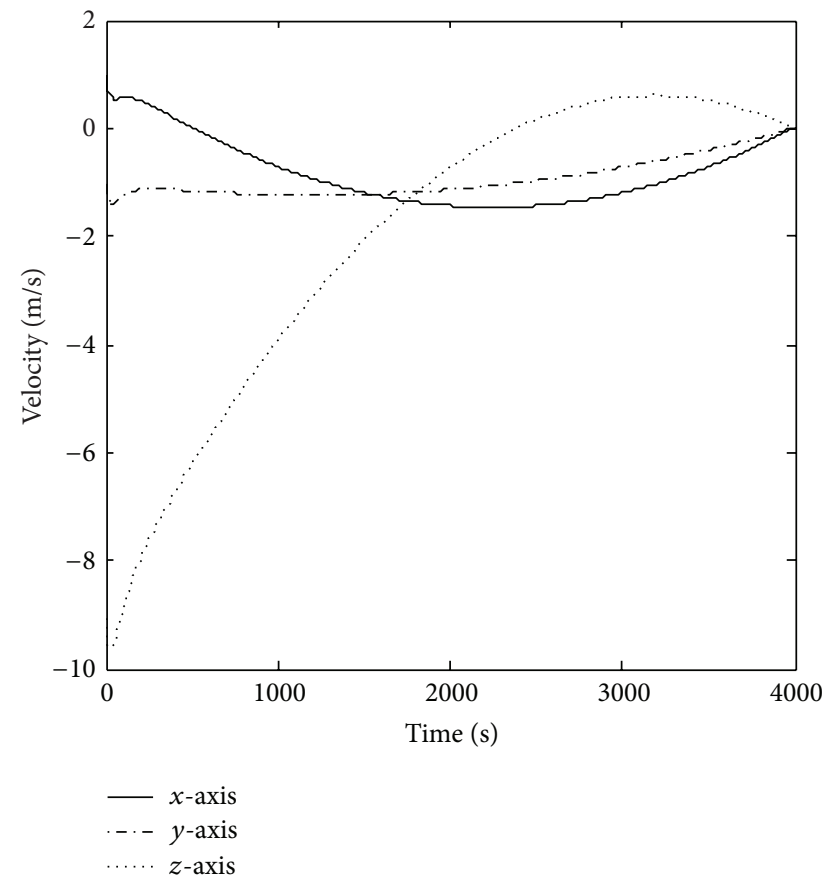

FIgURE 4: Descending velocity over time.

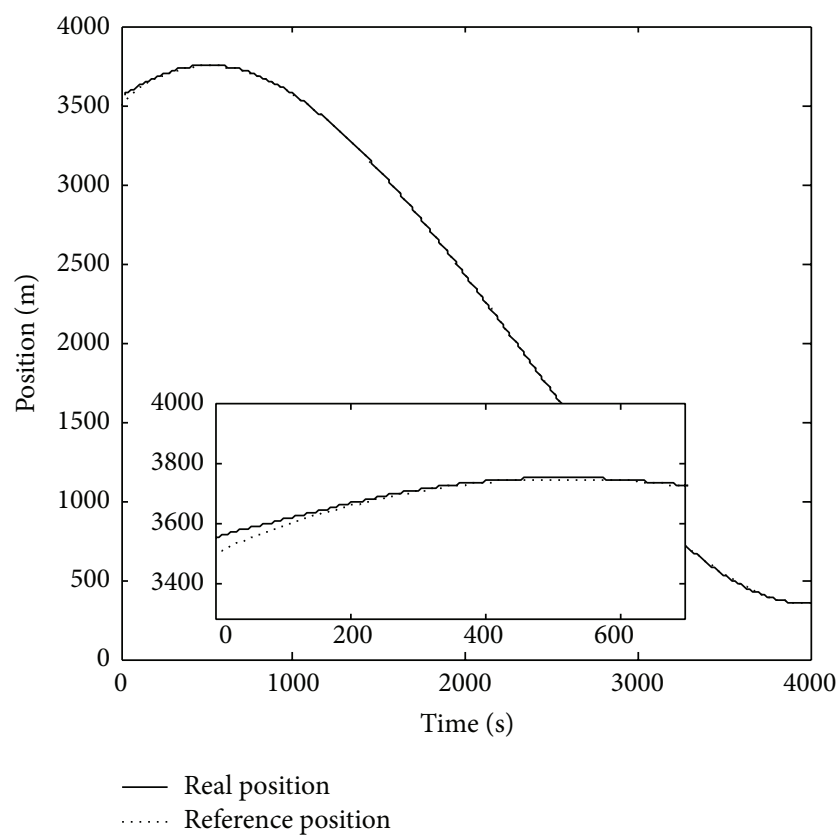

FIgURE 5: Descending position tracking of $x$-axis.

of soft landing on asteroids. The acceleration curve over time in landing phase is shown in Figure 12, which describes that the acceleration can rapidly converge to zero. The quality change of the probe during the landing phase is shown in Figure 13. With fuel consumption of the engine, the quality of

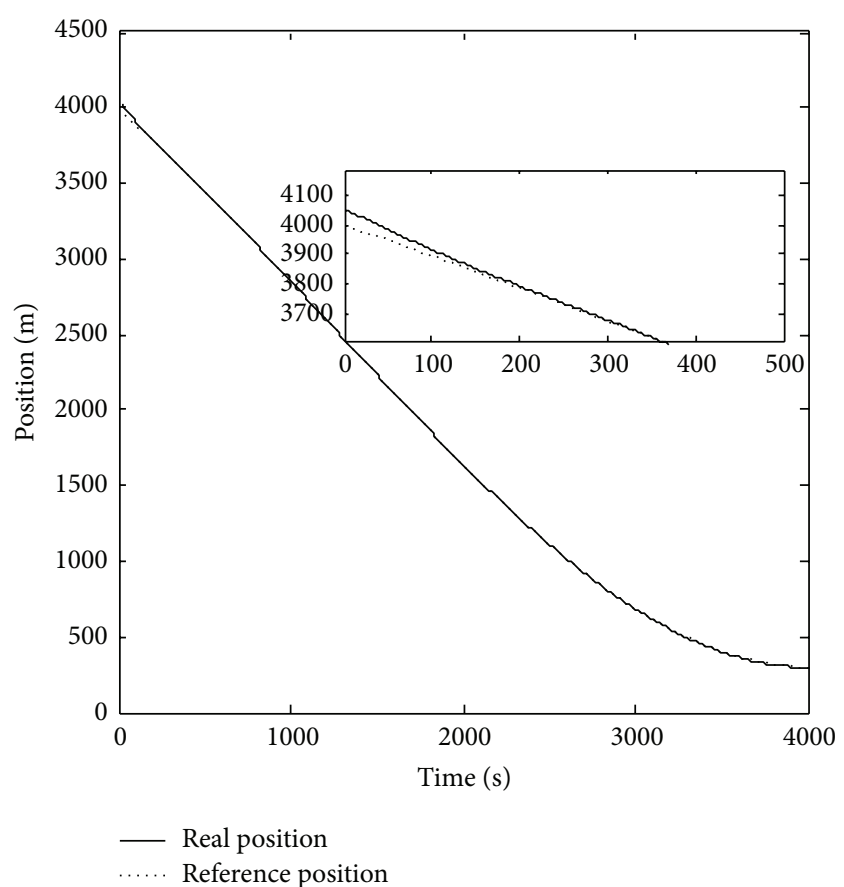

FIGURE 6: Descending position tracking of $y$-axis.

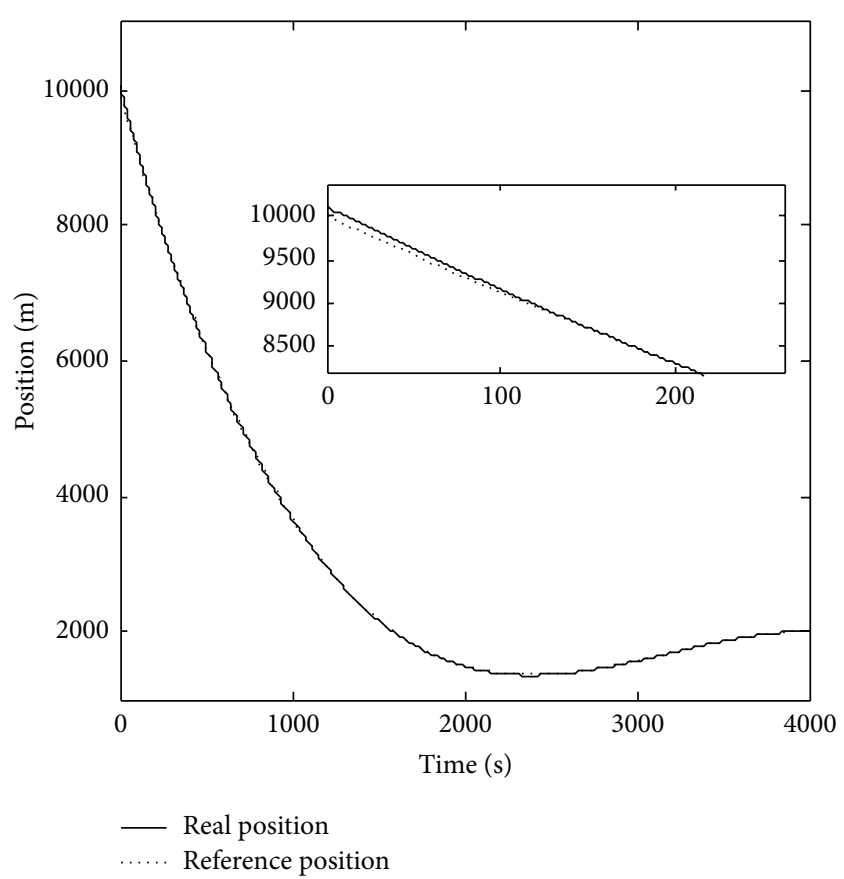

FIGURE 7: Descending position tracking of $z$-axis.

the probe is decreasing and ultimately maintains a fixed value during landing phase. With the strong robustness, the system has not been influenced on the disturbance caused by quality changes. Therefore, the probe could land smoothly and meet engineering requirements. 


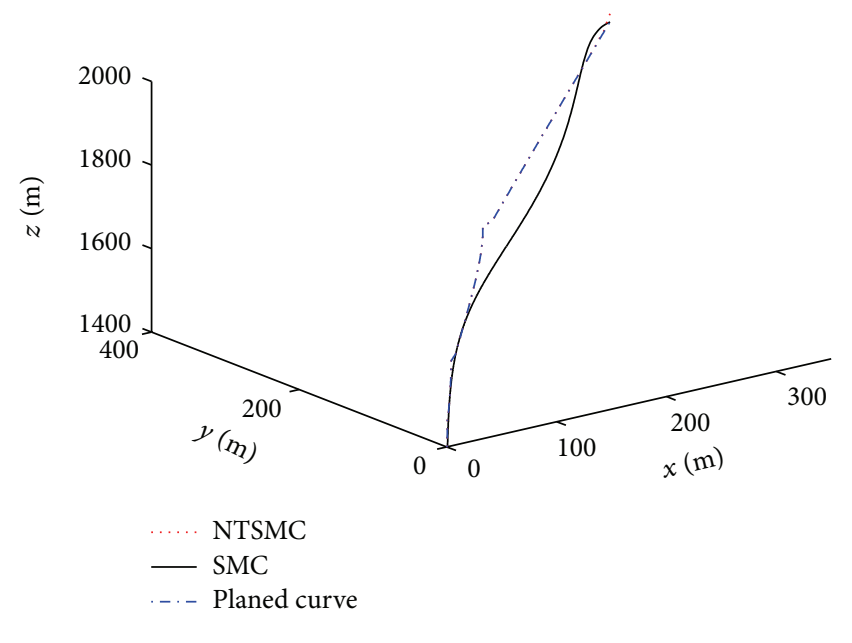

FIGURE 8: Landing trajectory curve.

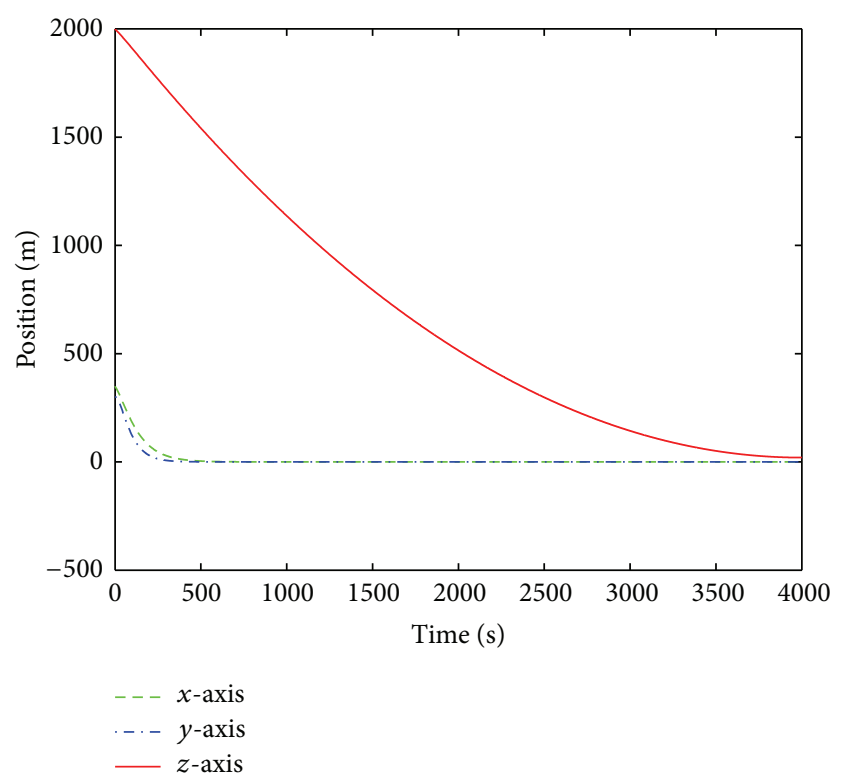

FIGURE 9: Landing position versus time.

\section{Conclusion}

In this paper, a continuous control law based on PD and the exponential reaching law of nonsingular terminal sliding mode control scheme is proposed to ensure the probe lands on asteroid safely and accurately. At first, the model of probe is deduced in the landing site coordinate system. With the suboptimal fuel consumption, the reference trajectory is planned. For soft landing on asteroid, PD and NTSMC control scheme are used in the descending and landing phase, respectively. Under PD control method, the probe can arrive at time landing position. Comparing with SMC control method, NTSMC has better performance, such as the quicker tracking speed, smaller error motion state, and less adjusting

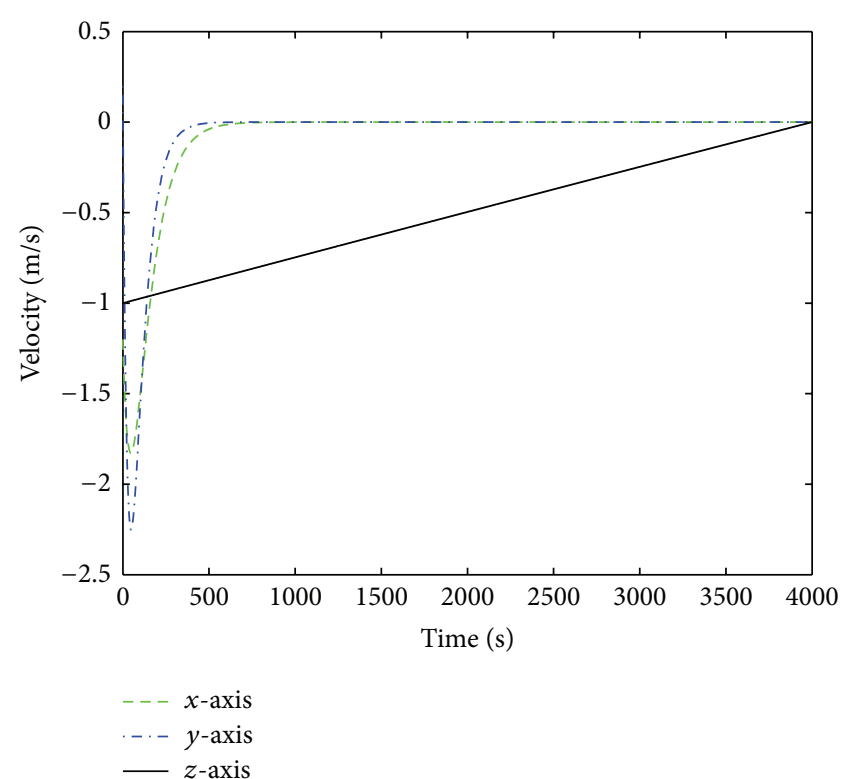

FIGURE 10: Landing velocity versus time.

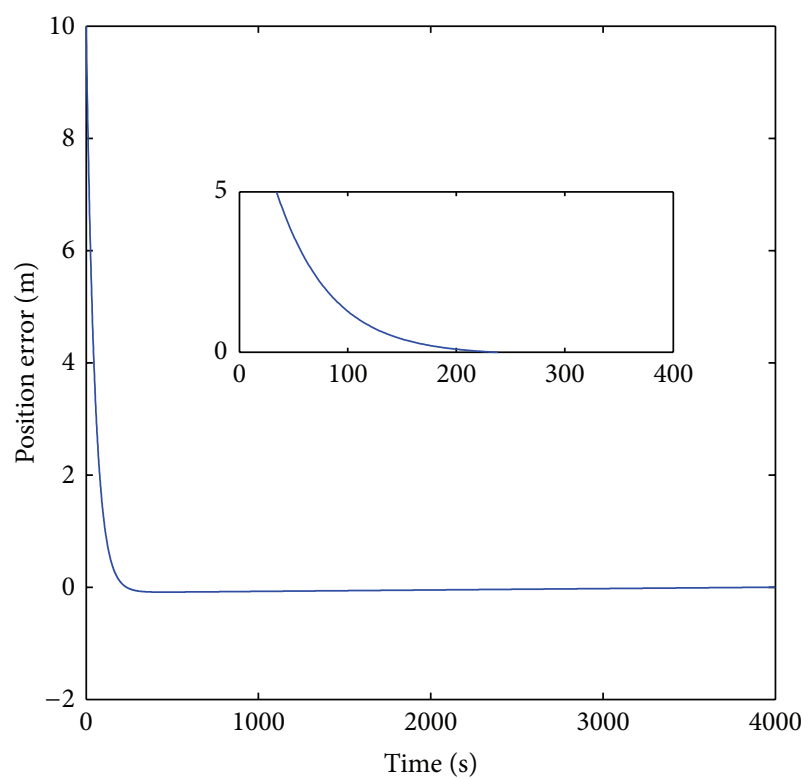

FIGURE 11: Landing position error of $z$-axis.

time, which can ensure the probe lands safely and accurately. Simulation results show that the position error of $z$-axis is less than $1 \mathrm{~m}$ and the final velocity is very small. Therefore, the feasibility of the continuous control law is verified.

\section{Conflict of Interests}

The authors declare that there is no conflict of interests regarding the publication of this paper. 


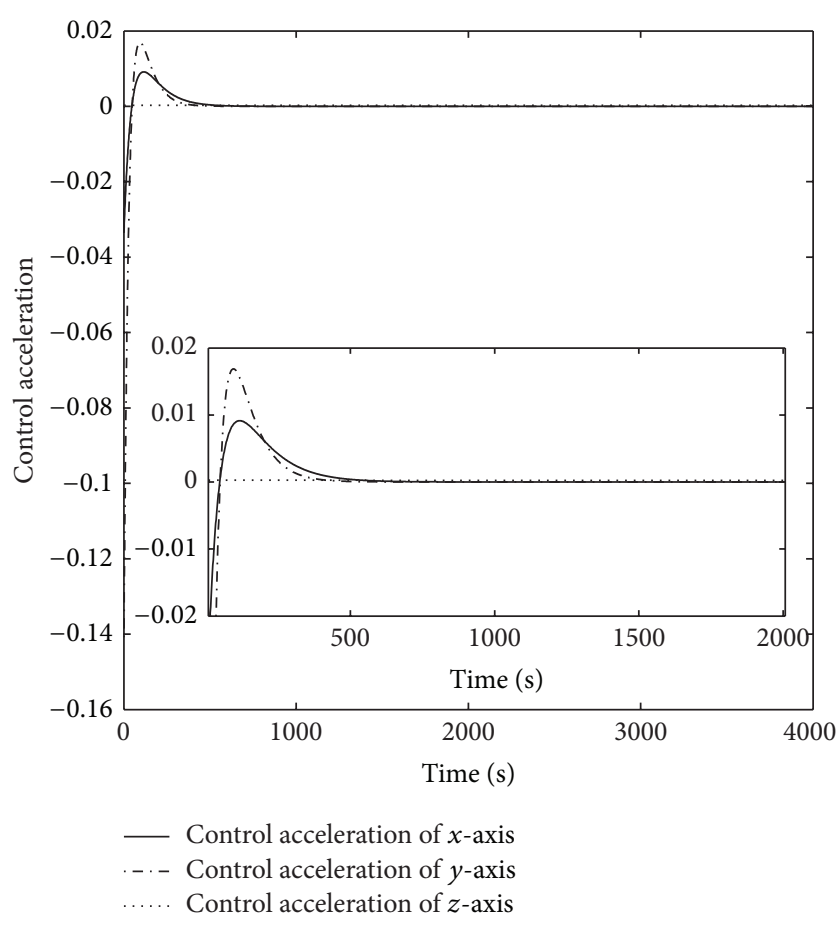

FIGURE 12: Landing acceleration.

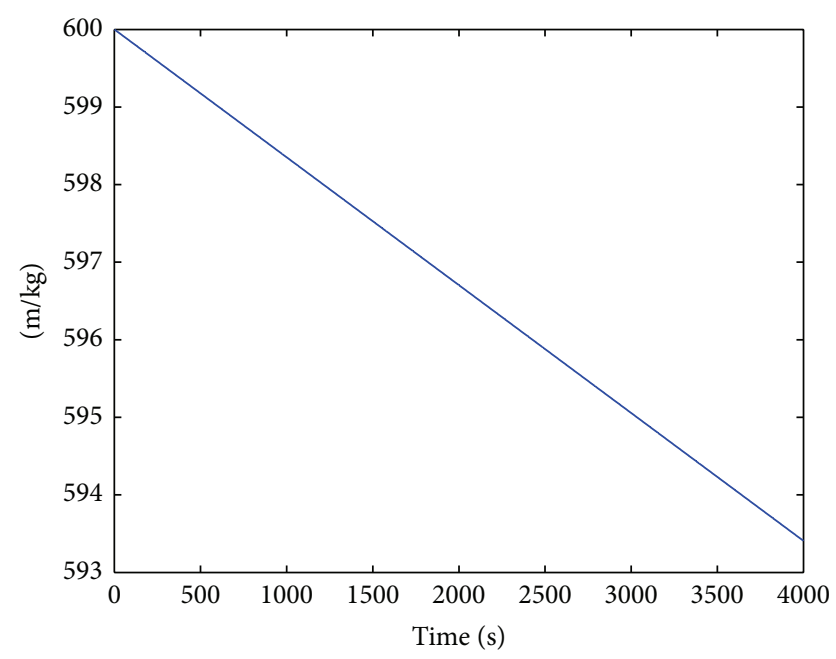

FIGURE 13: Quality curve of the probe.

\section{Acknowledgments}

Thanks are due to the support of National Basic Research Program of China (973 Program, 2012CB720000) and the National Natural Science Foundation of China (61403044).

\section{References}

[1] D. W. G. Sears, C. C. Allen, M. S. Bell et al., "The Hera nearEarth asteroid sample return mission: science requirements of the sample collector," Advances in Space Research, vol. 34, no. 11, pp. 2276-2280, 2004.
[2] J. Bellerose, A. Girard, and D. J. Scheeres, "Dynamics and control of surface exploration robots on asteroids," in Optimization and Cooperative Control Strategies, vol. 381 of Lecture Notes in Control and Information Sciences, pp. 135-150, Springer, Berlin, Germany, 2009.

[3] M. E. Holdridge, "NEAR shoemaker spacecraft mission operations," Johns Hopkins APL Technical Digest (Applied Physics Laboratory), vol. 23, no. 1, pp. 58-70, 2002.

[4] J. Veverka, B. Farquhar, M. Robinson et al., "The landing of the NEAR-Shoemaker spacecraft on asteroid 433 Eros," Nature, vol. 413, no. 6854, pp. 390-393, 2001.

[5] T. Kubota, T. Hashimoto, S. Sawai et al., "An autonomous navigation and guidance system for MUSES-C asteroid landing," Acta Astronautica, vol. 52, no. 2-6, pp. 125-131, 2003.

[6] R. Varatharajoo and R. Kahle, "A review of conventional and synergistic systems for small satellites," Aircraft Engineering and Aerospace Technology, vol. 77, no. 2, pp. 131-141, 2005.

[7] T. Misu, T. Hashimoto, and K. Ninomiya, "Optical guidance for autonomous landing of spacecraft," IEEE Transactions on Aerospace and Electronic Systems, vol. 35, no. 2, pp. 459-473, 1999.

[8] W. Zhang and J. Wang, "Nonsingular terminal sliding mode control based on exponential reaching law," Journal of Control and Decision, vol. 27, no. 6, pp. 909-913, 2012.

[9] H.-T. Cui and P.-Y. Cui, "Autonomous navigation and guidance for soft-landing asteroid," Journal of Astronautics, vol. 23, no. 5, pp. 1-5, 2002.

[10] S. Li, P. Cui, and H. Cui, "Autonomous navigation and guidance for landing on asteroids," Aerospace Science and Technology, vol. 10, no. 3, pp. 239-247, 2006.

[11] R. S. Felix Patron, R. M. Botez, and D. Labour, "New altitude optimisation algorithm for the flight management system CMA-9000 improvement on the A310 and L-1011 aircraft," Aeronautical Journal, vol. 117, no. 1194, pp. 787-805, 2013.

[12] T. L. Grigorie, R. M. Botez, A. V. Popov, M. Mamou, and Y. Mébarki, "A hybrid fuzzy logic proportionalintegral-derivative and conventional on-off controller for morphing wing actuation using shape memory alloy Part 1: morphing system mechanisms and controller architecture design," Aeronautical Journal, vol. 116, no. 1179, pp. 433-449, 2012.

[13] T. L. Grigorie, R. M. Botez, A. V. Popov, M. Mamou, and Y. Mébarki, "A hybrid fuzzy logic proportionalintegral-derivative and conventional on-off controller for morphing wing actuation using shape memory alloy Part 2: controller implementation and validation," The Aeronautical Journal, vol. 116, no. 1179, pp. 451-465, 2012.

[14] D.-C. Liaw, C.-C. Cheng, and Y.-W. Liang, "Three-dimensional guidance law for landing on a celestial object," Journal of Guidance, Control, and Dynamics, vol. 23, no. 5, pp. 890-892, 2000.

[15] D.-C. Liaw and C.-C. Cheng, "Variable structure control scheme for landing on a celestial object," International Journal of Systems Science, vol. 32, no. 3, pp. 295-301, 2001.

[16] S. B. Broschart and D. J. Scheeres, "Spacecraft descent and translation in the small-body fixed frame," in Proceedings of the AIAA/AAS Astrodynamics Specialist Conference and Exhibit, pp. 396-409, 2004.

[17] W. Shao, P.-Y. Cui, and H.-T. Cui, "Physical properties calculation of small body using points triangulations," Journal of Harbin Institute of Technology, vol. 42, no. 5, pp. 687-691, 2010. 
[18] A.-M. Zou and K. D. Kumar, "Adaptive output feedback control of spacecraft formation flying using chebyshev neural networks," Journal of Aerospace Engineering, vol. 24, no. 3, pp. 361372, 2011.

[19] S. Li, S. Ding, and Q. Li, "Global set stabilisation of the spacecraft attitude using finite-time control technique," International Journal of Control, vol. 82, no. 5, pp. 822-836, 2009.

[20] P. D. Washabaugh and D. J. Scheeres, "Energy and stress distributions in ellipsoids," Icarus, vol. 159, no. 2, pp. 314-321, 2002.

[21] R. A. Werner and D. J. Scheeres, "Exterior gravitation of a polyhedron derived and compared with harmonic and mascon gravitation representations of asteroid 4769 Castalia," Celestial Mechanics and Dynamical Astronomy, vol. 65, no. 3, pp. 313344, 1996.

[22] Z. Zexu, W. Wang, L. Li et al., "Robust sliding mode guidance and control for soft landing on small bodies," Journal of the Franklin Institute, vol. 349, no. 2, pp. 493-509, 2012.

[23] Y. Shan, Research on guidance problem of lunar probe for soft landing [Ph.D. thesis], Harbin Institute of Technology, 2009.

[24] X. Xi, G. Zeng, X. Ren, and H. Zhao, Lunar Probe Track Design, National Defense Industry Press, Hong Kong, 2001.

[25] H. Xiangyu, C. Hutao, and C. Pingyuan, "An autonomous optical navigation and guidance for soft landing on asteroids," Acta Astronautica, vol. 54, no. 10, pp. 763-771, 2004.

[26] Y. Feng, X. Yu, and Z. Man, "Non-singular terminal sliding mode control of rigid manipulators," Automatica, vol. 38, no. 12, pp. 2159-2167, 2002. 


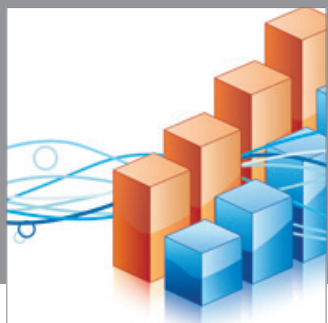

Advances in

Operations Research

mansans

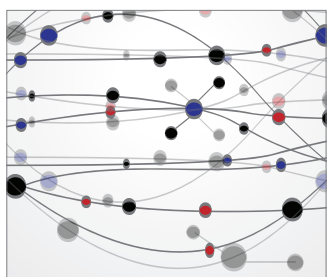

The Scientific World Journal
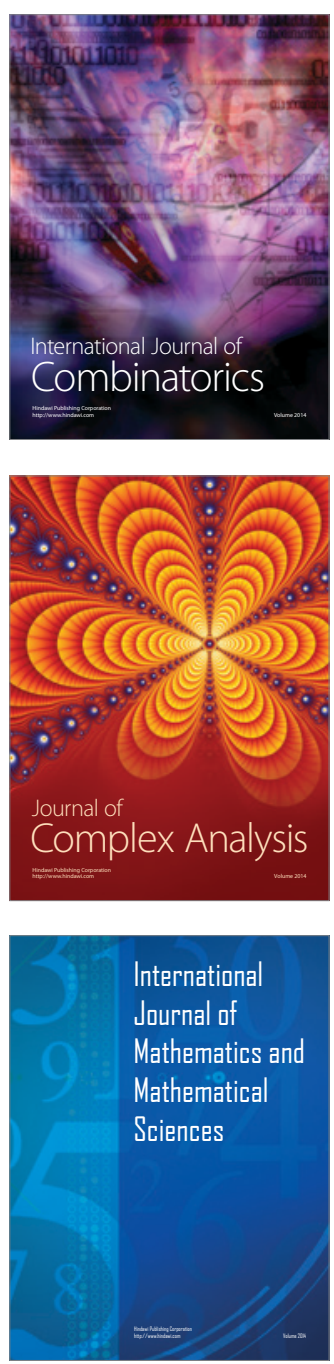
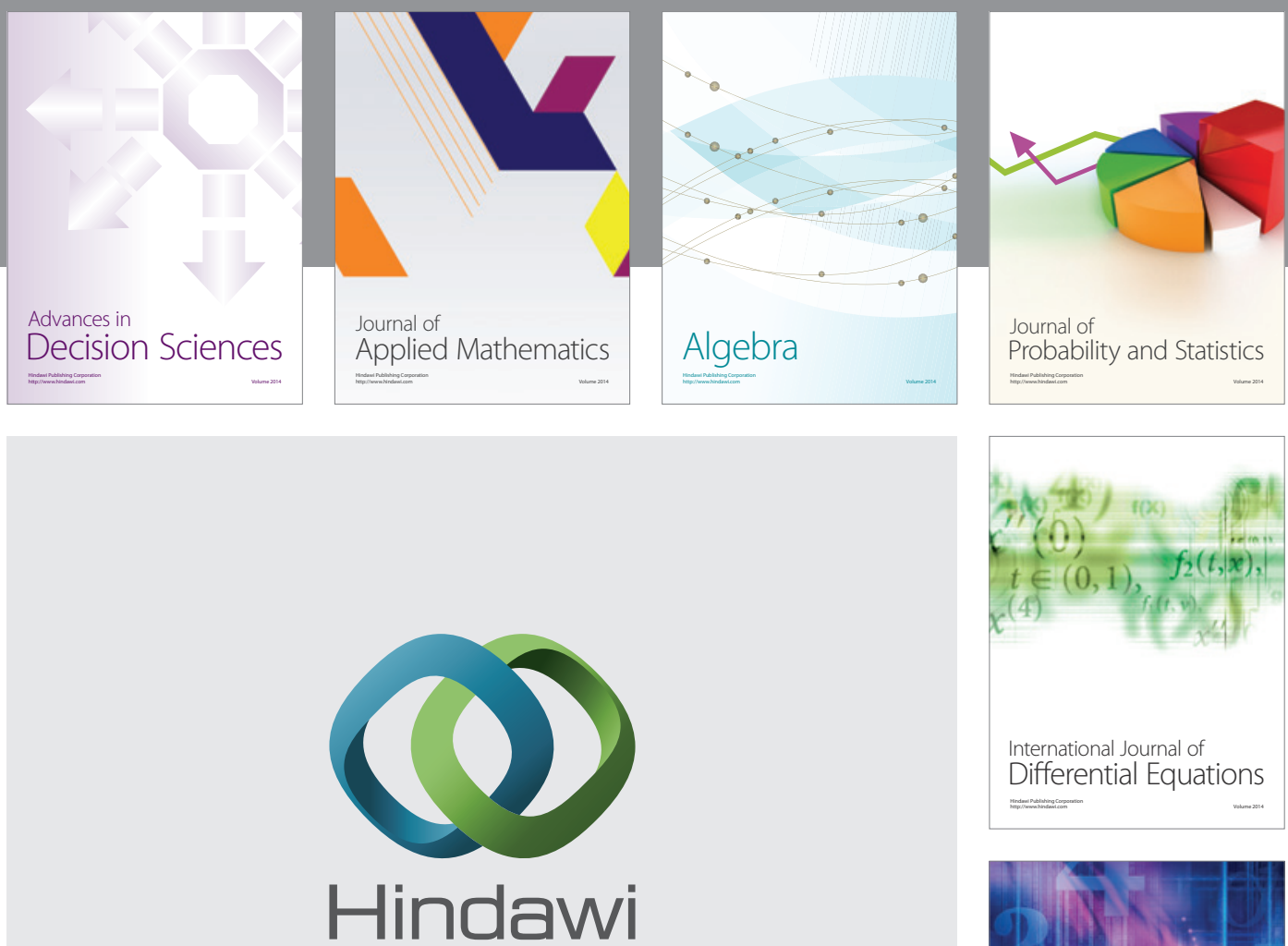

Submit your manuscripts at http://www.hindawi.com
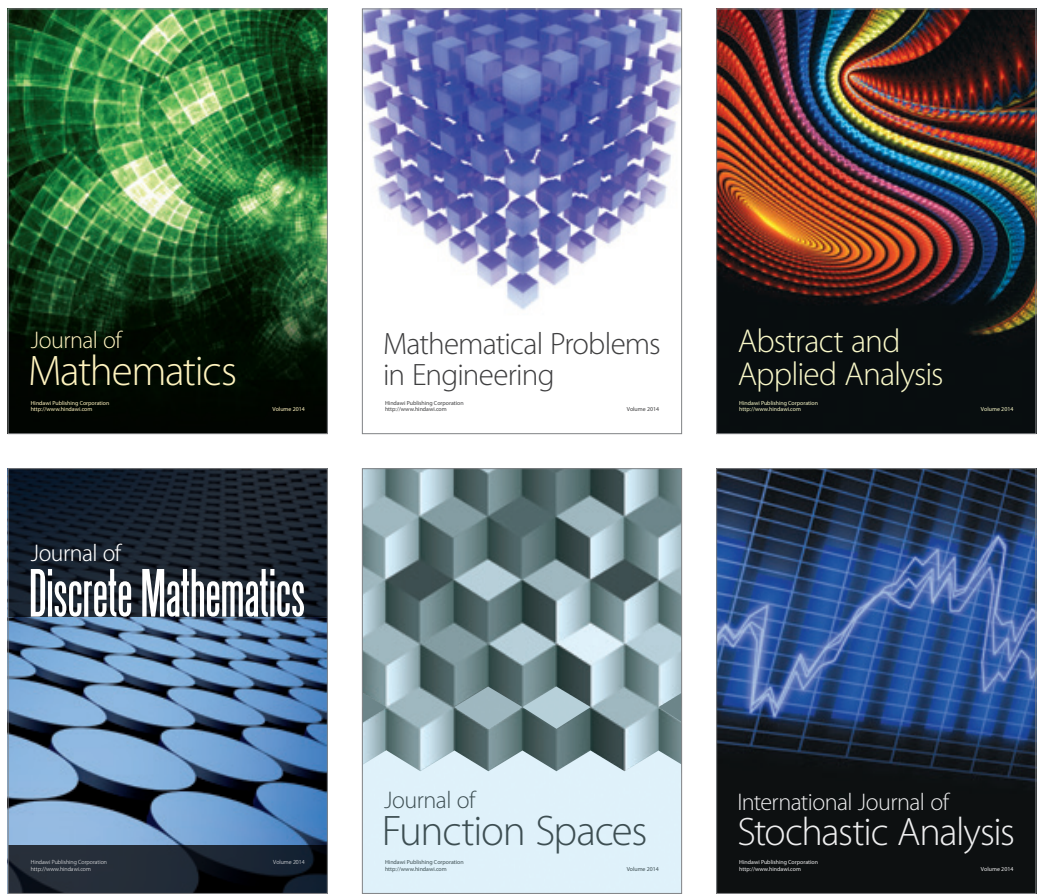

Journal of

Function Spaces

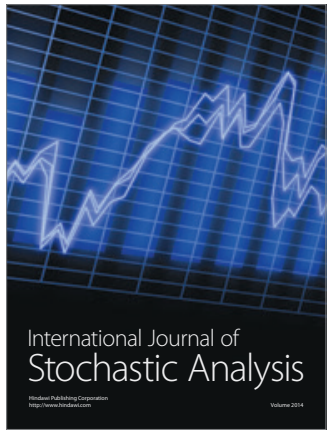

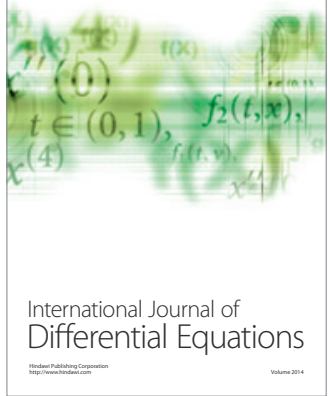
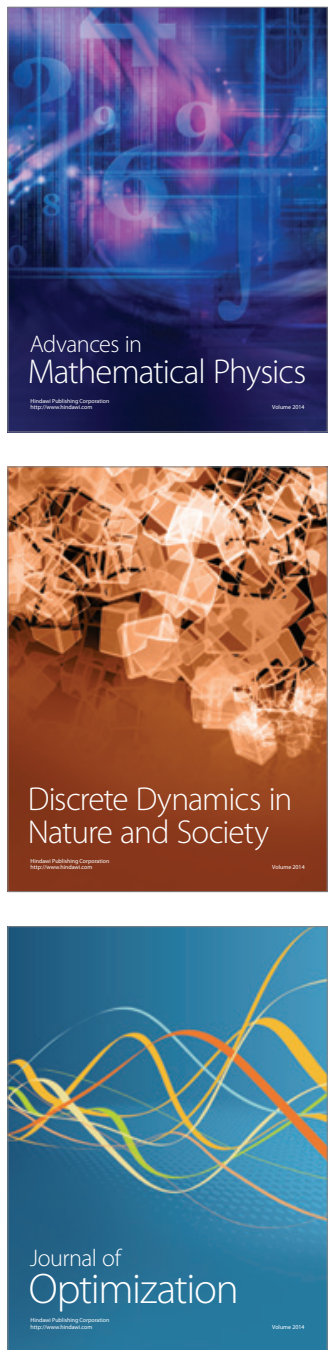